\title{
Study on the Analytical Method of High-speed Railway Noise Sound Field Simulation
}

\author{
Wang XiaoQin \\ Mechanical and electrical engineering college, Lanzhou Jiaotong University, \\ LanZhou, Gansu, 730070, China \\ 39681966@qq.com
}

\begin{abstract}
On the basis of the traditional structure of noise barrier, this paper seeks aerodynamic load to reduce the variation of maximum surface configuration (porous structure). Using the numerical simulation method, this paper builds a three-dimensional numerical model of deloading type noise barriers and trains. The large fluid calculation software, Fluent, is adopted to conduct simulation calculation of the high-speed deloading noise barriers. This thesis, conducting researches and analysis of the high-speed railway noise barrier aerodynamic load characteristics on the surface, concludes the characteristics of loading surface pressure distribution, time-history features as well as the law of influence factors, which is applicable to deloading sound barrier and lays a theoretical foundation for deloading sound barrier designing.
\end{abstract}

Keywords: High-speed railway, noise sound field, simulation, sound barrier

\section{Introduction}

With the rapid development of high-speed trains, the speed has been constantly upgraded and many issues have become increasingly prominent, which influences the train development [1-3]. Research shows that high-speed train aerodynamic noise volume are mainly influenced by the speed of the train, $U$, and the aerodynamic noise radiation power is proportional to about $\mathrm{U}^{6}$ to $\mathrm{U}^{8}$. Therefore, the aerodynamic noise will rise dramatically while the train speed is rising. When the running speed reaches $300 \mathrm{~km} / \mathrm{h}$, the aerodynamic noise will exceed the wheeltrack noise and will become the main source of noise [4-5]. With higher train speed, railway noise pollution is also rapidly increasing. Excessive noise will seriously affect physical and psychological status of passengers and people living near tracks as well as their daily life. What's more, noise may also cause fatigue failure on equipment and surrounding buildings and shorten their service life. Thus the highspeed train noise has become an urgent issue. As an important vehicle, sound barriers have become been widely used to reduce acoustic noise. The inhibition effect mainly depends on sound barriers height and the distance between sound barriers and trains. The higher the sound barriers are and the shorter the distance between trains, the noise reduction effect is better. Therefore, from the perspective of reducing noise, it is better to have higher sound barriers and shorter distance. However, at that moment, the impact of the transient airflow caused by running train on sound barriers is very strong. Here are two main contents to be considered when designing sound barriers: 1 . noise reduction effect of noise barriers and 2 . The safety of sound barriers while the aerodynamic load is working. Deloading noise barriers are proposed to be concept products on the basis of comprehensive consideration of those two aspects. Two methods can be adopted to reduce the aerodynamic load on noise barriers, one is elastic connection in basic parts, the other is trepanning on a noise barrier plate. Based on the basic theory of 
aerodynamics, this thesis uses numerical simulation method, builds the deloading type noise barrier and three dimensional numerical models of trains, adopts large fluid calculation software Fluent to conduct simulation calculation of the high-speed train deloading process and conducts researches and analysis on aerodynamic load characteristics to conclude the law of influences exerted by sound barrier aerodynamic load schedules, space distribution characteristics and sound barrier height on sound barrier aerodynamic loading features.

\section{Aerodynamic Noise Characteristics of High-speed Trains}

In terms of geometry characteristics, noise sources can be divided into point sources, line sources and surface sources. High-speed railways are usually electric railways with high running speed and a large number of viaducts. In addition to wheeltrack noise from middle and low speed railways, high-speed railway noise also has a large portion of aerodynamic noise and collector system noise. According to research materials [6], the wheeltrack noise is directly proportional to the square of the train speed. The aerodynamic noise and collector system is directly proportional to the power of the train speed. When the train speed is higher than $300 \mathrm{~km} / \mathrm{h}$, aerodynamic noise and the collector system noise has become the main body of noise. Compared with SD railways, high-speed railways have higher position of noise sources and further distance of dissemination. Due to higher proportion of high-speed railway viaduct structure, the secondary structure of the noise has become an important part of the high-speed railway noise.

China adopts weighting pressure level equivalence A to evaluate noise influences. Acoustic frequency response of A meter is equivalent to the sensitivity of ear and broad band sounds. It has strong attenuation in low-frequency stage and weak attenuation in high-frequency stage. In large attenuation to low frequency, attenuation smaller at high frequencies. For noise with fluctuating or inconsistent sound levels, consistent equivalence $\mathrm{A}$ is adopted, which is equal to the $\mathrm{A}$ level of $\mathrm{T}$ (identical time interval) and continuous stable noise as well as fluctuating noise energy. Its mathematical expression is:

$$
L_{\text {Aeq }, T}=10 \lg \left[\int_{t_{2}-t_{1}}^{1} \int_{t_{1}}^{t_{2}} p_{A}^{2}(t) d t\right]
$$

Here, ,LAeqT represents equivalent sound pressure level meter right $\mathrm{A} d \mathrm{~dB}(\mathrm{~A}) ; \mathrm{t} 2$-t 1 refers to time interval T; PA is weighting sound pressure A (PA) and P0 refers to benchmark acoustic pressure $(\mathrm{Pa})$.

If measure in identical sampling time interval, a series of A sequences can be concluded. The A sound level data during $\mathrm{M}$ can be calculated as following:

$$
L_{e q}=10 \lg \left[\frac{1}{T} \sum_{i=1}^{N} 10^{0.1 L_{A i}} \tau_{i}\right]
$$

Here, LAi refers to the ith A weighting pressure level dB (A); T refers to the total measuring time (s); ${ }^{\tau_{i}}$ is the sampling time interval (s) and $\mathrm{N}$ represents the number of testing data.

When running at a high speed, the train body surface and wheeltrack will produce very complex side flow, which refers to a non-lineal and multi-scale fluid motion, irregular in space and time. This motion, together with irregular fluid border produces complex flowing status. This kind of complexity is usually represented in randomness, rotating and statistics [7]. At present, the numerical simulation of flow calculation methods generally fall into three categories: Direct Simulation (DNS), 
Large Eddy Simulation (LES) and Reynolds-averaged Navier-Stocks equations (RANS) [8].

The Direct Numerical Simulation is aimed to get all the parameters in the flow field by solvbing N-S equations. Reynolds Time-Average Parameter Method can be adopted as the movement of flow still follows the general law of mechanics and continuum movement characteristics from the perspective of $\mathrm{N}-\mathrm{S}$ equation of incompressible flow so as to infer the Reynolds equation based on the time average field. Two commonly used equation models are $\mathrm{k}-\mathrm{s}$ equations and formal $\mathrm{k}$ $\varepsilon$ equation model:

$$
\begin{gathered}
u_{t}=\rho C_{u} \frac{k^{2}}{\varepsilon} \\
\varepsilon=\frac{u}{\rho} \overline{\left(\frac{\partial u}{\partial x}\right)}=C_{D} \frac{k^{3 / 2}}{l}
\end{gathered}
$$

Here, $\mathrm{Cu}$-empirical constant; $\varepsilon_{\text {is }}$ the turbulent kinetic energy dissipation rate.

The basis of Large Eddy Simulation is the calculation method of turbulent flow spiral embellish algorithm for learning design. The general idea of this simulation method is to filter the flow in the filed by LES filter of instantaneous flow movement. The rest of large flow motion directly uses N-S equations. And small flow motion that have been filtered are moving through the calculation of attached equations. According to the basic thought of LES, it is necessary to adopt a method to distinguish large flows and small flows. Different from RANS, Les uses the space average method and the variable $u$ is decomposed into ui in N-S and time grid variable u. Leonard's formula can be used as:

$$
u_{i}(x)=\int_{-\infty}^{+\infty} G\left(x-x^{1}\right) u\left(x^{1}\right) d x^{1}
$$

Here, $\mathrm{G}(\mathrm{x}-\mathrm{x}$ ') is the filter function. Commonly used filter functions are gaussian functions and cap functions. Simple cap functions are widely used:

$$
G\left(x-x^{1}\right)= \begin{cases}1 / \Delta & \left|x-x^{1}\right| \leq \Delta / 2 \\ 0 & \left|x-x^{1}\right|>\Delta / 2\end{cases}
$$

Here, $\Delta$ is the average time grid, three dimensional time $\Delta=\Delta_{1} \Delta_{2} \Delta_{3}$ are time grids in 3 different directions: $\mathrm{X}, \mathrm{y}, \mathrm{z} . \Delta \rightarrow 0$, LES transfers into DNS.

\section{The Numerical Analysis Model of High-speed Train Aerodynamic Load Noise Barrier}

Calculation Model: This paper uses three CRH2 marshalling trains to be respectively the first car, the middle and the last one. The length of train is $77.4 \mathrm{~m}$, the height is $3.7 \mathrm{~m}$, the width is $3.4 \mathrm{~m}$ and the length of barriers is $50 \mathrm{~m}$. Simplified measures are shown as follows: 1) simplify some irregular structures such as bogies, pantographs, etc.; 2) omit tracks and some structures and conduct seamless processing on trains and grounds; 3) although the sound barrier structure is relatively simple, but the pillar structure still needs to be omitted. The surface needs to be seamless and smooth and the sound barrier needs to be long and straight. Simplified structure model and the high speed train noise barrier model are shown in the figure below:

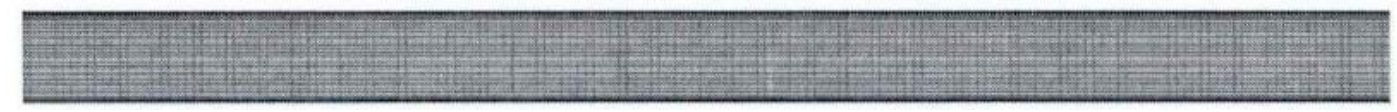

(a) Sound Barrier Structure Model 


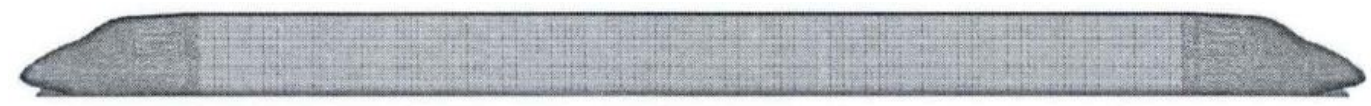

(b) High-speed Train Structure Model

Figure 1. Simplified Sound Barrier and High-speed Train Model

The distance between the calculation boundary of high-speed train flows and the train model should surpass 7-10 times of the model height. When 3-4 times is taken, the error is less than 5\%, which is within the acceptable range. Therefore, the flow field calculation is $300 \mathrm{~m} * 50 \mathrm{~m} * 3 \mathrm{~m}$. According to the characteristics of the passing process of noise barrier, this paper divides the computational domain into sound barriers, regions away from the noise barrier, train movement area and areas away from the train. The calculation area is shown in the figure below.

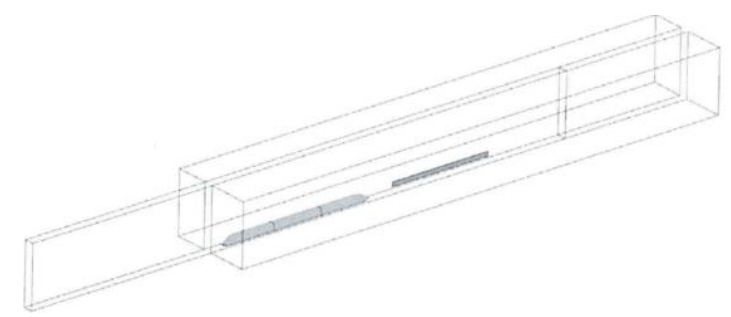

Figure 2. Model Calculation Area

Analysis of Grids and Sensitivity: This paper adopts GAMBIT software to conduct grid division of high-speed train passing through the filed outside sound barriers. GAMBIT software can generate structural grid, non-structural grid and hybrid grid, etc. This software has a better adaptability to elaborate or generalize grid points, or generate variable grid and sliding grid, etc.

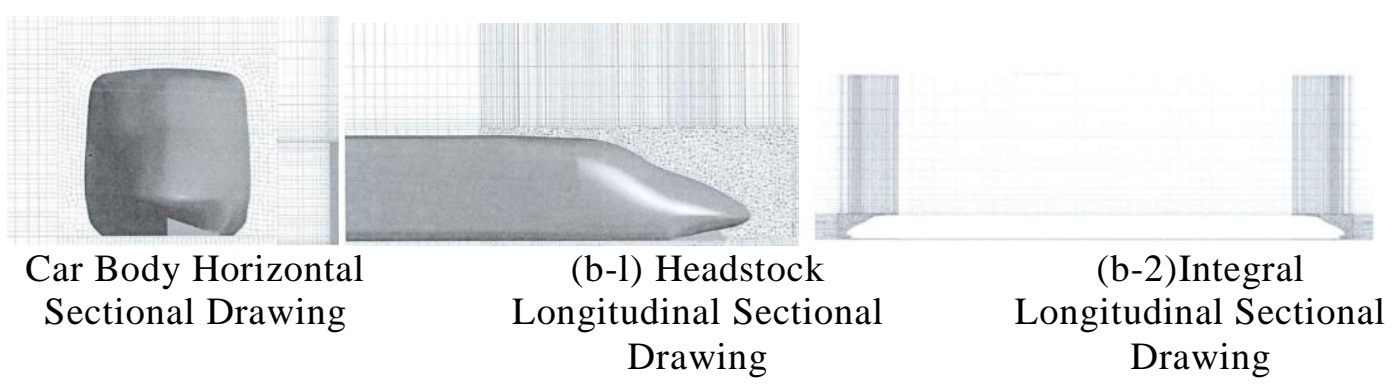

Figure 3. Flow Field Grid Sectional Drawing (Surrounding Part)

In accordance with the calculation model in this paper, as sound barrier is far away from sound barrier field, and the model is regular, the structural grid is adopted. While the train moving field, due to the train working, only adopts nonstructural grids on the headstock and tailstock. Other areas adopt structural and nonstructural grids, figure 3 is the horizontal and longitudinal sectional drawing in flowing areas.

This paper has carried out the grid sensitivity analysis. Continuous improvement of grid density and a large amount of calculation finally determine the whole flow field area unit is about 2.3 million. As shown in table 1 below, under the condition of different number of grid cells, when the train passed the sound barrier at the speed of $350 \mathrm{~km} / \mathrm{h}$, there are some pressure peak values and relevant errors from the middle observation site, which is $6 \mathrm{~m}$ away from the entrance of the sound barriers. From the table, it is obvious to see that when the grid cell number is less than 2.3 
million, the pressure peak value of observation site is bigger; when the grid cell number is more than 2.3 million, the pressure peak value scarcely changes. Thus, we can determine the appropriate grid cell number is 2.3 million.

\section{Table 1. Grid Sensitivity Analysis and Contrast}

\begin{tabular}{|l|l|l|}
\hline Grid Cell Number(Ten Thousand) & Pressure Peak Value(Pa) & Error(\%) \\
\hline 230 & 660.3 & \\
\hline 180 & 749.6 & 13.52 \\
\hline 200 & 696.4 & 5.47 \\
\hline 240 & 659.2 & 0.17 \\
\hline 250 & 658.9 & 0.21 \\
\hline 300 & 657.7 & 0.39 \\
\hline
\end{tabular}

Initial Conditions and Boundary Conditions: Under initial conditions, inlets and outlets both adopt standard air medium, when the initial velocity is zero and the pressure is an atmospheric pressure. This paper studies the passing process of highspeed trains in an open flow field, thus, the boundary conditions for inlets and outlets are not speed boundaries but constant pressure boundary conditions. The back boundary is the inlet boundary, the front is the outlet and both inlets and outlets are the atmospheric pressure as shown in Figure 4. Wall boundary flow is common to encounter, which is used to restrict fluid and solid field. In the simulation calculation in this paper, grounds of three flow fields are all supposed to wall conditions, while the surface and sound barrier of fast-moving train surface, compared with air movement, exert important influences on the external flow fields, which is the wall effect. Thus, suppose the boundary condition of high-speed train and sound barriers as solid wall boundaries as shown in Figure 5.

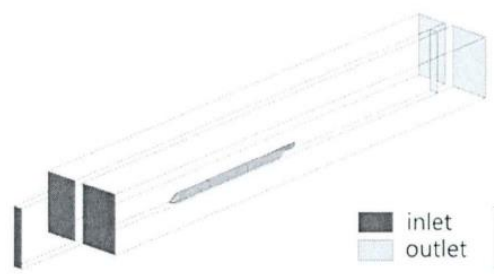

Figure 4. Pressure Boundary Condition

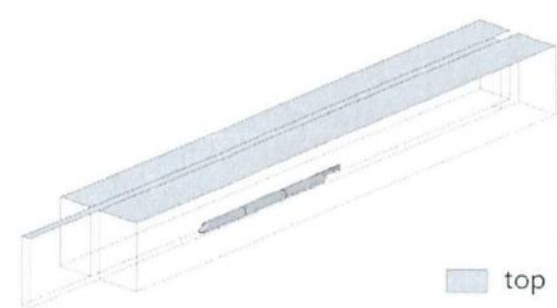

Figure 6. Symmetric Boundary Conditions

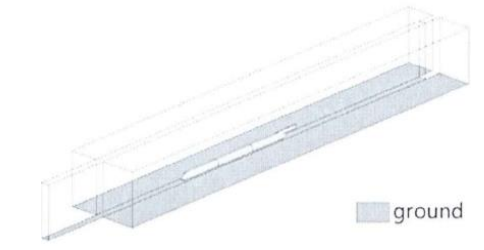

Figure 5. Wall Boundary Conditions

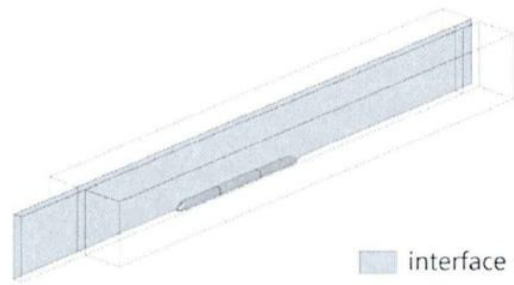

Figure 7. Mutual Boundary Conditions

This thesis adopts symmetric boundary conditions at top boundaries in three calculation fields and reduces the calculation times as shown in Figure 6. The method is used to define interfaces of different unit areas and those interfaces simulate relative motion by mutual boundaries. This paper sets up mutual boundaries between high-speed trains and sound barriers and boundaries between 
trains and outflow field. Date from different calculation fields can be exchanged and transferred by interfaces as shown in figure 7 .

Observation Position on the Surface of Sound Barriers: Select eight points as observation points as shown in figure 8 . From the bottom up at the entrance, set 3 observation points $\mathrm{P} 1, \mathrm{P} 2$ and $\mathrm{P} 3$. The distance from sound boundaries on the horizontal direction is 6 meters; $\mathrm{P} 1$ is 0.2 meters away from the ground; P3 is 0.1 meters away from the top of the barrier, P2 is half the height of the sound barrier. From the bottom up three points are P4, P5 and P6; From the bottom up layout three observation points is $\mathrm{P} 7, \mathrm{P} 8$ and $\mathrm{P} 9$, which is symmetric to the sound barriers.

Train run direction

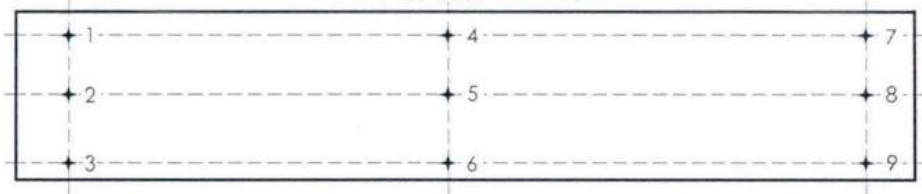

Figure 8. Sound Barrier Observation Distribution

\section{Deloading Sound Barrier Aerodynamic Value Simulation and Verification}

The sound barrier is $3.4 \mathrm{~m}$ away from the central line of railway and the speed is $350 \mathrm{~km} / \mathrm{h}$, the sound barrier height is $2.15 \mathrm{~m}$, the thickness of $0.175 \mathrm{~m}$, the length is $50 \mathrm{~m}$. The high-speed train marshalling has three quarters with the length of $77.4 \mathrm{~m}$, width $3.38 \mathrm{~m}$ and height of $3.7 \mathrm{~m}$. Take the point $20 \mathrm{~m}$ from acoustic barrier to entry as the start. While the train entering into the sound barrier, the influence of headstock waves and tailstock waves pass the barrier. The positive and negative pressure field is shown in figure 9. In terms of the headstock pressure field, the maximum positive pressure on the windward surface is about $666 \mathrm{~Pa}$ and the maximum negative pressure is about -674 pa. In terms of the tailstock pressure field, the maximum positive pressure on the windward surface is about $471 \mathrm{~Pa}$ and the maximum negative pressure is about $-601 \mathrm{~Pa}$. Headstock wave and tailstock wave can influence $50 \mathrm{~m}$ length and two sound barriers are both under positive and negative wind pressure field, which is moving with the train. The wind pressure volume is reducing from the bottom to the top in the pressure field.

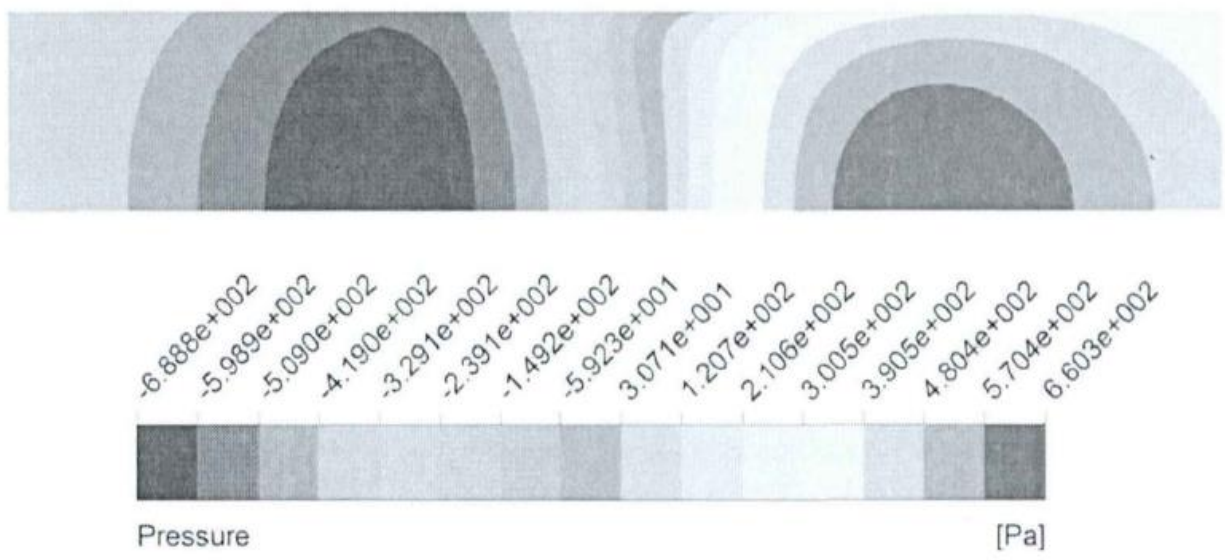

Figure 9. Sound Barrier Surface Positive and Negative Pressure Field 


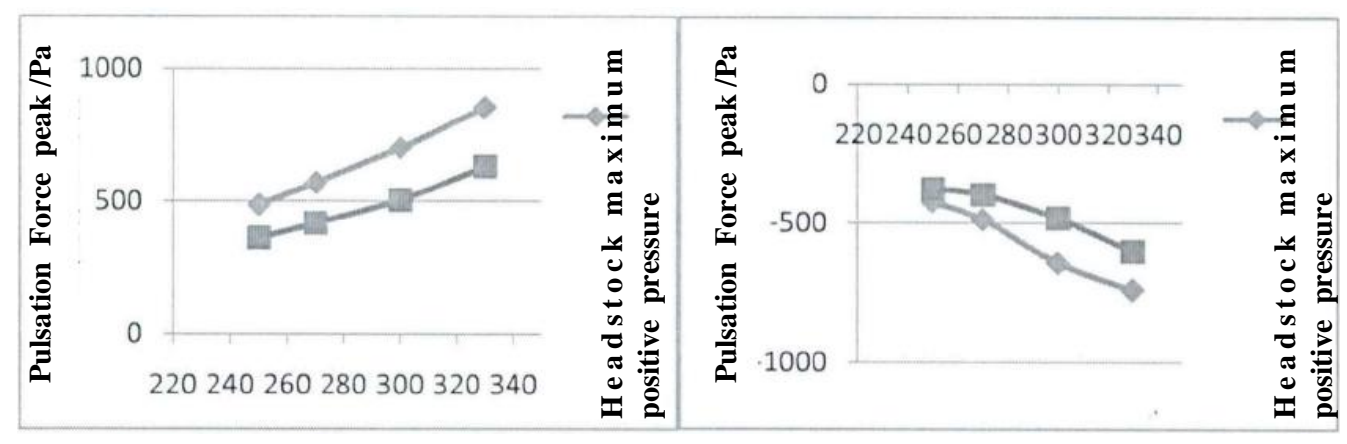

Figure 10. 3.15m Relations of Sound Barrier Intermittent Force Summit Volume between Speed

It can be seen from Figure 10 that the higher speed the high-speed train runs, the greater the headstock and tailstock summit volumes are. The train speed changes from $250 \mathrm{~km} / \mathrm{h}$ to $330 \mathrm{~km} / \mathrm{h}, 1.32$ times that of the original and the pulse peak power is 1.75 times larger. Thus we can include that speed is the main factor to influence sound barrier aerodynamic load. The sound barrier structure mechanics performance design should take the maximum train speed pulse condition of dynamic load caused by design. As the speed increases from $250 \mathrm{~km} / \mathrm{h}$ to $330 \mathrm{~km} / \mathrm{h}$, the absolute value of positive and negative pressure increases from $64 \mathrm{~Pa}$ to $112 \mathrm{~Pa}$ and the tailstock absolute value increases from $24 \mathrm{~Pa}$ to $28 \mathrm{~Pa}$. The absolute value of the headstock is greater than the one of the tailstock. According to the figure, we can conclude that the equation is directly proportional to the square of the train running speed of linear fitting curve: $\mathrm{P}=0.1003(7,3.6) / 2$. Figure 11 shows the biggest difference that the train speed is $270 \mathrm{~km} / \mathrm{h}$, and the simulation calculation results is $556.4 \mathrm{~Pa}$, but the fitted curve is $564.2 \mathrm{~Pa}$ with an error of $1.4 \%$. Therefore, when the center line of the orbit is $3.4 \mathrm{~m}$ away from the distance and CIIH2 passes the $3.15 \mathrm{~m}$ barrier, the maximum pressure absolute value can be concluded by above equation in a more accurate way.

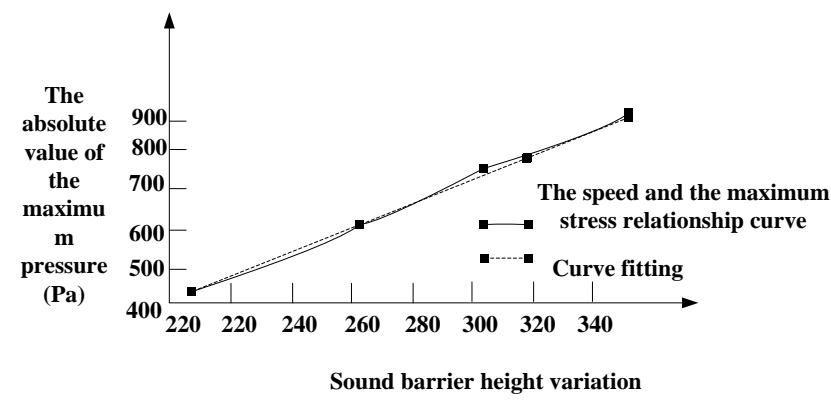

Figure 11. The Speed and the Maximum Stress Relationship Curve

When the high-speed train passes through the sound barrier, the flow fields are basically identical in terms of shape. Compare the effects of different height of barriers exerted on the same observation. The heights are $2.1 \mathrm{rn}, 2.5 \mathrm{~m}>3.15 \mathrm{~m}$ and $3.5 \mathrm{~m}$, and the curve can be seen. 


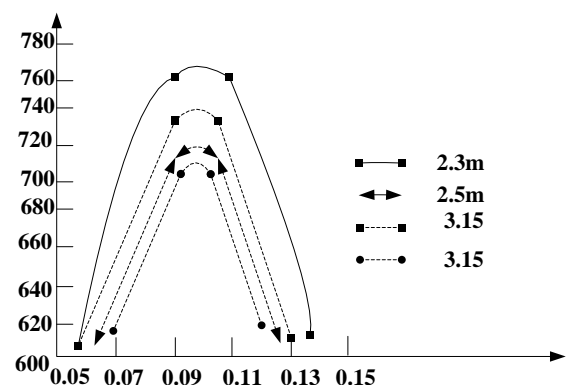

\section{Figure 12. Influence Exerted by Height of Value of Barrier on Intermittent Force}

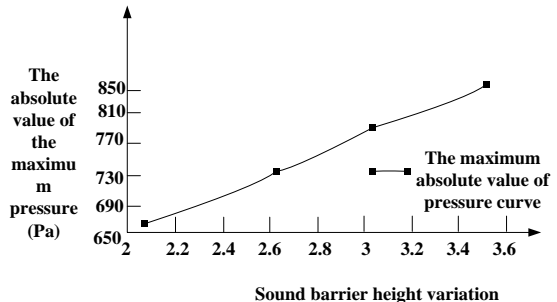

Figure 13. The Maximum Absolute Pressure Curve

\section{Conclusion}

Results and Discussion: According to the figure, we can see that the pressure increases while the height is going up. That is because the increasing height of barriers limits the air in the bottom to expand to other palaces. While the pressure goes down, the aerodynamic loading is increasing. Figure 12 and figure 13 shows the maximum absolute value of pressure curve. It can be seen that the sound barrier height increases from $2.1 \mathrm{~m}$ to $3.5 \mathrm{~m}$, the peak power increases $179 \mathrm{~Pa}, 1.27$ times larger. The increase of noise barrier height increased under the pulse peak power of noise barrier. The increase of height causes larger peak values. And the increasing height also bring increasing bending moment. Thus, under the condition of reducing noises, the low sound barriers design can be a better choice.

\section{Acknowledgements}

It should contain the information regarding any research grant support or the assistance of colleagues.

\section{References}

[1] H. Liu and S. Lu, "Calculation Methods of High-Speed Train Noise [J]", Communication and Transportation Engineering Journal, vol. 2, no. 2, (2002), pp. 41-44.

[2] J. Zhu and J. Jing, "High-Speed Train Noise Research and Control [M]", Railways and Vehicles, vol. 48, no. 5, (2011), pp. 1-8.

[3] S. Zhang, "350km *h-1 High Speed Train Noise", Sound Identification and Control [J]", Science of China's Railway, vol. 90, no. 1, (2009), pp. 86-90.

[4] X. Yang, Y. Gao, Y. Cheng and F. Liu, "High-speed Train Aerodynamic Noise Analysis [J]", Noise and Vibration Control.

[5] F. Yang, L. Zheng and P. He, "Value Simulation of High-speed Train Aerodynamic Noise [J]", Textbooks for Computing, vol. 19, no. 1, (2010), pp. 44-47.

[6] H. Yin, Y. Li and X. Gu, "Analysis on High-speed Railway Sound Barrier Effects and Factors [J]", China's Railway, vol. 12, (2009), pp. 45-46.

[7] S. Lin, "Researches on Friction Noise on Surface of High-speed Trains [D]", Hangzhou: Zhejiang University, (2011).

[8] D. Ma, "Manual for Noise and Vibration Controlling [M]", Beijing: China Machine Press, vol. 9, (2002), pp. 90-93, 31-32.

[9] H. Liu, "Researches on High-speed Railway Sound Barrier Aerodynamic Loading and Connecting Structure [D]", Changsha: Central South University, (2012).

[10] D. Li, S. Zhou and G. Hongye, "380km/h High-speed Train Aerodynamic Loading Simulation Analysis [J]”, Railway Building, vol. 9, (2011), pp. 133-136. 


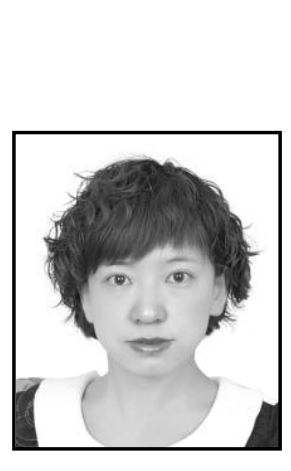

\section{Author}

Wang XiaoQin, She is currently a teacher, lecturer and Associate professor.

Current position, grades: Teacher, lecturer

University studies: Mechanical and electrical engineering college, Lanzhou Jiaotong University

Scientific interest: Aerodynamics

Publications $<$ number or main $>: 15$

Experience: Doctoral students in reading, once worked in Lanzhou Jiaotong University, and had rich experience. 
International Journal of Signal Processing, Image Processing and Pattern Recognition Vol.8, No.8 (2015) 